\title{
Expected asteroseismic performances with the space project PLATO
}

\author{
Mariejo Goupil ${ }^{1}$ and the WP120 ${ }^{2}$ Members \\ ${ }^{1}$ Observatoire de Paris, LESIA, Observatoire de Paris, PSL Research University, CNRS, Université Pierre et Marie Curie, Univer- \\ sité Paris Diderot, 5 place JUles Janssen 92190 Meudon, France \\ ${ }^{2}$ PLATO STEllar and Seismology Inferences workpackage ${ }^{\star}$
}

\begin{abstract}
The PLATO (PLAnetary Transits and Oscillations of star) space project will observe about fifty percents of the sky with the main purpose of detecting, confirming and characterizing transiting exoplanets of (super)Earth sizes in the habitable zone of solar-like stars. Determining masses, radii and ages of exoplanets require the knowledge the masses, radii and ages of the host stars. We give a brief presentation of the main features of the mission. We then discuss some expected seismic performances of PLATO for characterizing bright solar-like stars, focusing on the challenging determination of accurate/precise stellar ages.
\end{abstract}

\section{Introduction}

The space project PLATO (PLAnetary Transits and Oscillations of stars) was selected as the M3 mission by ESA on February 2015. The mission is currently in phase B2 (consolidated definition phase). The adoption process is planned for February 2017 for a launch in 2025 by a Soyouz rocket (L2 orbit). The main scientific objective of PLATO is to answer the following questions

1. How do planets and planetary systems form and evolve?

2. Is our Solar System special or are there other systems like ours?

3. Are there potentially habitable planets?

In order to answer the above questions, the PLATO mission must :

- detect and characterize the (super) Earth-type planets, that-is determine the bulk properties (mass, radius, mean density) of planets in a wide range of systems, including terrestrial planets in the habitable zone of solar-like stars.

By (Super)Earth-like planets, one means planets with masses, $M_{p}$, and radii, $R_{p}$, such that $M_{p} \leq 1 M_{E}$; $R_{p} \leq 2 R_{E}$ where $M_{E}$ and $R_{E}$ respectively are the Earth mass and radius. The habitable zone is defined here as the region around a star where liquid water on the surface of an Earth-like planet is in principle possible.

It must be stressed that the space mission Kepler (and K2) does - and the future space mission TESS will mostly provide transiting planets with orbital periods smaller than 80 days that-is planets with orbits not further than a Mercury-like orbit. Only PLATO will be

${ }^{\star}$ http://www.oact.inaf.it/plato/PPLC/PSM/Entries/2010/12/19_ STELLAR SCIENCE.htm able to provide a significant number of (super) Earthtype planets in the habitable zone of solar-like stars (an Earth-size planet at about 1 au from its host star).

The expected output of PLATO is $\sim 100-400$ precisely characterized exoplanets including 5 - 30 (super)Earths in the habitable zone of solar-like stars.

- explore the diversity of the planets and planetary systems

In order to determine accurately the mass and radius of a planet, one needs to determine with the same accuracy the mass and radius of the host star.

- study the evolution of planets and planet systems with age.

The age of the planet is assumed to be the age of the host star.

- study the internal structure of stars and how it evolves with age.

The accuracy/precision on the mass, radius and age determinations of the host stars depend on the quality of the stellar models (numerical precision, degree of realism of the physical description)

The above issues define the PLATO core programme. In addition, a complementary science programme is included. It is complementary because it has no influence on the design of the mission but will widely contribute to the output of the mission with a large number of astrophysical applications. To name a few, seismology of massive stars clusters, studies of galactic populations. This can be extended to any astrophysical open question which needs high-precision, continuous time series of photometric measurements for a large sample of stars. 
The PLATO management consortium (PMC) is led by the Principal Investigator, H. Rauer (Germany). It includes more than 350 researchers from fourteen ESA Member States, as well as an Associate Member state, and several other nations across the world. The structure of the PLATO consortium is made of three main branches the payload, the PLATO Data Center (PDC) and the PLATO Science Management (PSM). The PLATO Data Center (PDC) is responsible for producing the data output of the mission. The PSM is in charge of specifying the algorithms ad tools which will be implemented and used by the PDC. The structure of the PSM is composed of several workpackages [Exoplanet science (WP110), Stellar science (WP120), Target/fields characterization (WP130), Follow up coordination (WP140), and Complementary Science (WP150)].

A full description of the Plato mission is detailed in [1] (see also PLATO Definition Study Report (2017)). We focused here on the expected performances of PLATO for the stellar part of the core program. The paper is organized as follows: Sect.2 explains how to reach the scientific objectives. Sect. 3 presents several main issues of the scientific preparation. Sect. 4 adresses some expected performances for the stellar part of the core programme. Sect.5 draws some conclusions.

\section{Reaching the PLATO scientific objectives}

Three techniques will be combined to reach the PLATO scientific objectives:

- Detection of exoplanets by photometric transits. This will provide the orbital parameters and the planet-tostar radius ratio $R_{p} / R_{*}$. The relative uncertainty on the planet radius must be smaller than $3 \%$ for the reference planet (an Earth-size planet orbiting at about 1 au from a GOV star with $V=10$ i.e. orbital period of about 1 year). A driver for the mission efficiency is therefore the number of bright $(V \leq 11)$ dwarfs and subgiants stars of spectral type F5-K7 which will be continuously monitored with a high signal-to-noise ratio $(\leq 34 \mathrm{ppm} / \mathrm{h})$ over at least two years time periods. One must observe at least $15000-20000$ such stars.

- Confirmation of the planet nature of the candidate by spectroscopic ground-based follow-up. Measurements of radial velocities will give the planet-to-star mass ratio $M_{p} / M_{*}$ for transiting exoplanets. The relative uncertainty on the reference planet mass, $M_{p}$, must be smaller than $10 \%$ for a host star with an apparent magnitude $V=10$. This will be obtain for the brightest planet candidates with the highest interest ranking.

- Precise seismic determinations of the mass, $M_{*}$, radius $R_{*}$ and age for the host stars.

The specifications for the exoplanet part require a noise-to-signal ratio $N S R \leq 80 \mathrm{ppm} / \mathrm{h}$ for detection and $N S R \leq 34 \mathrm{ppm} / \mathrm{h}$ for the planet radius measurements for stars with $V \leq 11$ and $2-3$ years of observation. The requirements for the host stars accordingly are that the relative uncertainties are no larger than $1-2 \%$ for the radius, $15 \%$ for the mass and $10 \%$ for the age of the reference star at magnitude 10. The reference star is defined as a G0V star with the solar mass and radius and an effective temperature of $6000 \mathrm{~K}$, see the document 'PLATO-DLRMIS-LI-002_i1.1.pdf').

The reference star and any solar-like stars are expected to vibrate with solar-like oscillations. The above requirements then translate into the following specifications : the oscillation frequency uncertainties of the solar-like stars must be of the order or smaller than $\sim 0.1-0.2 \mu \mathrm{Hz}$ for frequencies around $v_{\max }$, defined as the frequency at maximum amplitude in the frequency domain. These specifications lead to further specifications which are that the noise-to-signal level must be of the order or smaller than $34 \mathrm{ppm} / \mathrm{h}$ for $V \leq 11$, the time series must be long and continuous with no periodic gaps of similar time scales than the oscillation ones.

\subsection{Payload, observing scenario and data product}

The payload is a multi-telescope concept for a wide-fieldof view for each pointing: 24 «normal» $12 \mathrm{~cm}$ cameras will collect white light $(500-1050 \mathrm{~nm})$ at a cadence $25 \mathrm{~s}$ for stars with apparent magnitude $8<V<16$. The fieldof-view is set to $48.5^{\circ} \times 48.5^{\circ}\left(2250 \mathrm{deg}^{2}\right)$. In addition, 2 «fast» $12 \mathrm{~cm}$ cameras with a $2.5 \mathrm{~s}$ cadence will observe in two colours (in a FoV $550 \mathrm{deg}^{2}$ (tot)) stars with apparent magnitude $4 \leq V \leq 8$. The daily downloaded telemetry will amount to about $435 \mathrm{~Gb}$. Note that several samples of stars will be observed with different numbers of telescopes corresponding to different noise levels at a given apparent magnitude.

The observing scenario (Fig. 1) is based on four years of observation after commissioning and the possibility of a two-year extension. The choices are either two long runs of 2 years each and a sky survey in a step-and-stare mode ( 2 to 5 months) during the extension period or a three-year long run and one year for a sky survey in a step-and-stare mode ( 2 to 5 months). The spacecraft will operate a $90^{\circ}$ rotation around the line-of-sight every 3 months so that the expected duty cycle is about $93 \%$.

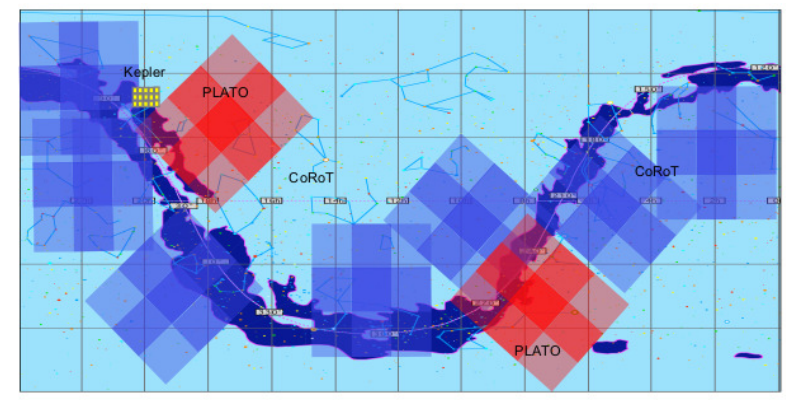

Figure 1: The reference observing scenario: 2 long runs and several short runs, covering $50 \%$ of the sky. 
Data product will be generated from the PLATO mission at different levels as follows:

- L0 : The validated light curves and centroid curves for all individual telescopes, housekeeping data, imagettes;

- L1 : The calibrated light curves and centroid curves for each star and corrected for instrumental effects e.g., jitter;

- L2 : Scientific output:

- The planetary transit candidates and their parameters with formal uncertainties;

- The asteroseismic mode parameters with formal uncertainties;

- The stellar rotation periods and stellar activity models inferred from activity-related periodicities in the light curves, with formal uncertainties;

- The seismically-determined stellar masses, radii and ages of stars, (and their formal errors), obtained from stellar model which oscillation frequencies fit best the observed ones;

- Living catalogue of confirmed planetary systems and their characteristics using light curves and time transit variations.

- Lg : Follow up ground-based observation data;

- L3 : Living catalogue of confirmed planetary systems fully characterized by combining information from the planetary transits, the seismology of the planet-hosting stars, and the results of the ground-based observations; This represents the final and highest level PLATO science deliverable.

\subsection{Samples of stars}

The set of target stars in the core programme is divided into four samples. With 24 telescopes and the current baseline observing strategy,

- the P1 sample includes about 15000 ( 20000) bright dwarfs and subgiants ( $V \leq 11)$ with spectral type F5-K7, a noise level $\leq 34 \mathrm{ppm} / \mathrm{h}$, long observing run (LOP) with a $25 \mathrm{~s}$ cadence. This sample will include the PLATO 'Rosetta stones';

- the P2 sample contains more than 1000 dwarfs and subgiants $(V \leq 8.2)$ (300 stars with 2 colours) with spectral type F5-K7, a noise level of $\leq 34 \mathrm{ppm} / \mathrm{h}$, LOP with a $2.5 \mathrm{~s}$ cadence. This sample will offer the possibility of caracterizing their atmospheres with ground (E-ELT) or space (JWST) facilities and the possibility of seismically caracterizing a large number of stars for improving the calibration and description of the stellar models;

- the P4 sample is made of more than $5000 \mathrm{M}$ dwarfs $V \leq$ 16, sampling time 25s, noise level $800 \mathrm{ppm} / \mathrm{h}$.

- the P5 sample consists of $\geq 245,000$ dwarfs and subgiants $(V \leq 13)$ with spectral type F5-K7 with a lower SNR than P1, a 25s cadence. This sample is subdivided in two sub-samples:

- P5-bright $(V \leq 11)$ stars for which mass measurements from ground will be possible;

- P5-faint $(11<V \leq 13)$ stars with a lower SNR but still with sufficiently precise characterization for statistical studies.

\section{Scientific preparation}

\subsection{Input catalogue and target selection}

Telemetry limitations impose the pre-selection of PLATO targets for the detection of planets. The optimal field selection is closely related to the target selection. The number of F5 or later spectral type dwarfs and sub-giants for which we can have photometry with the required SNR must be as large as possible. This led the WP130 workpackage to build a PLATO Input Catalogue (PIC) which includes P1P5 targets, and provides their main parameters. The PIC is built using already existing star catalogues. As an illustration, this count yields for the fields of the reference observing scenario about 13000 stars populating the P1 sample, about 29000 stars in the P5 sample with $V \leq 11$, $34 \mathrm{ppm} / \mathrm{h}<N S R \leq 80 \mathrm{ppm} / \mathrm{h}$; about 80000 stars for in the P5 sample with $V<13, N S R<80 \mathrm{ppm} / \mathrm{h}$. Each step -and-stare can provide about 6100 stars in the $\mathrm{P} 1$ sample and about 160000 stars in the P5 sample.

The Gaia release catalogues will also provide wellclassified nearby dwarf/sub-giant stars to optimise the content of the PIC.

\subsection{Estimation of planet candidates and needed resources for ground-based follow up}

Over the whole space part of the PLATO mission, one expects the detection of about 13000 transiting planets of all sizes and periods out of which about 3400 transiting planets with host stars bright enough $(V \leq 11)$ suitable for radial velocity follow up. From this set, the current counts foresee 2200 planets with radius $\leq 2 R_{E}$ and which could be rocky in nature. These estimates by the WP110 workpackage are based on the stellar populations in the PIC and our current knowledge of the exoplanet planetary systems.

After identification of a planet candidate with PLATO data, ground-based follow up observations are needed to confirm and characterise the planetary nature of the companion. For most planet candidates, accurate masses will be derived from spectroscopic radial velocity observations. The PLATO mission therefore includes organized ground-based follow-up observations which will be carried out by the Ground-based Observations Programme (GOP) team. The needed ground-based resources considered two steps : first, observations must be designed to detect false positives and confirm the planet nature of the candidate. Second, the mass of the planet must derived from ground-based spectroscopic measurements. The necessary amount of resources has been estimated by the WP140 workpackage and found to be comparable to a large ESO programme. Then at least 150- 200 superEarths are expected to be characterized out of which more than several dozen of planets are expected to seat in the 
habitable zone of their host stars, representing the main targets for PLATO. The last figure remains however quite uncertain since it depends on the small planet occurrence rates in the habitable zone, a poorly known quantity that PLATO will contribute to establish on more solid grounds.

\subsection{Plato noise level as a function of magnitude and number of telescopes}

For performances studies, the stellar signal in the noise-tosignal ratio, NSR, is assumed constant. The PLATO noise is made of several components : the photon noise of the target star, the random noise from the instrument and the residual systematic noise from the instrument after correction. The residual systematic noise from the instrument after correction must not be larger than $1 / 3$ of the total random noise. At this step of the performance studies, the signals considered as noises either for exoplanet or seismic studies (stellar activity, granulation, oscillatory components, transit effects in a power spectrum) are ignored.

The noise-to-signal ratio, SNR, for the reference star and for 24 telescopes was estimated by the performance working group on the basis of our knowledge of the instrument performance today and is found to be $\sim 25 \mathrm{ppm} / \mathrm{h}$ at $V=10$.

\section{Expected performances for the stellar part of the core programme}

For seismic performance estimations, only studies focusing on the P1 sample have been carried out so far [2]. The estimated number of stars passing the PLATO specifications is $\sim 15000$ out of which more than $\sim 5000$ stars will be seismically characterized with relative uncertainties $\Delta A / A \sim 10 \%, \Delta R / R \sim 2 \%, \Delta M / M \leq 15 \%$ where $A$, $R_{\star}$ and $M_{\star}$ respectively mean the age, the radius and the mass of the star [3].

Assuming the PLATO noise level mentioned in the previous section and the case of the reference star, verification of the performances must then be carried out with two successive steps:

- step 1: reaching oscillation frequency uncertainties down to $\Delta v_{n, l} \sim 0.2 \mu \mathrm{Hz}$ for several frequencies around $v_{\max }$

- step 2: assuming the step 1 frequency uncertainties, one must obtain the uncertainties for the stellar parameters at the level of $2 \%$ (radius), $15 \%$ (mass), $10 \%$ (age) for the reference star at magnitude $V=10$.

Calculations show that the radius and mass uncertainties easily satisfy the PLATO specifications with the tools and methods currently used today for seismically characterizing CoROT and Kepler stars. On the other hand, the age specification is a challenge. From now on, we therefore focus on the age specification.

Age uncertainties can be seen as arising from several sources. One source is the propagation of the observational frequency uncertainties as estimated in step 1, we call it below age random error. A second source is referred to as systematic error and arises because the age must be determined with the use of stellar models which are not perfect. The physics implemented in evolutionary codes to compute stellar models remain by many aspects uncertain. Some processes such as convective energy transport are modelled with free parameters which values are currently set empirically. This can introduce large biases in stellar age derivation, everything else being equal [4]. Improvement in the modelling of these processes as well as modelling of missing processes are still in development and not yet implemented in stellar evolutionary codes. In the error budget, one must also include the impact of observational uncertainties on the non seismic constraints used in the stellar modelling such as the effective temperature, the surface gravity, the metallicity and when available the luminosity.

In the remaining of this section, we report first on the expected age random error for the reference star belonging to the P1 sample. A second part discusses the expected age systematic error for a star belonging to the P1 sample. For this second part, hare and hounds exercises ,(hereafter HH), were carried out over 2014-2015 within the workpackage WP120 (or STESSI for STEllar Seismic Inferences) using Plato simulated light curves.

\subsection{Age random error for the reference star belonging to the $\mathrm{P} 1$ sample}

As mentioned above, we estimate first the frequency uncertainties and second the age uncertainties that arise from the frequency uncertainty propagation for the reference star.

Step 1 : we use the [5]'s relation to compute the frequency uncertainties for the reference star.

$$
\sigma(v)=\left[f(\beta) \frac{\Gamma}{4 \pi T_{o b s}}\right]^{1 / 2}
$$

in $H z$ where $f(\beta)=(1+\beta)^{1 / 2}\left[(1+\beta)^{1 / 2}+\beta^{1 / 2}\right]^{3}$ and $\beta=N S R$ is the noise-to-signal ratio, $\Gamma$ (in $\mathrm{Hz}$ ) the width of the mode and $T_{o b s}$, the observing time (in s).

This relation is valid for radial modes $(l=0)$. For nonradial modes, one must take into account the mode visibility and the inclination angle. The validity of the Libbrecht'relation is well established [6]. Nevertheless, we also checked its validity with the results of the PLATO $\mathrm{HH}$ exercises (not shown here).

The target is the reference star with an apparent magnitude $V=10$ and the observing time, $T_{o b s}$, is assumed to be 2 years. We evaluate the frequency uncertainties at $v_{\max }$ i.e. $\sigma\left(v_{\max }\right)$ which then involves $\beta_{\max }=N S R_{\max }$ at $v_{\max }, \Gamma$ the width of the modes at $v_{\max }$ and $N S R_{\max }=$ $B_{\max } /\left(V_{\ell} H_{\max }\right)$ where $H_{\max }$ is the mode height for radial modes at $v_{\max } . B_{\max }$ is the PLATO for the reference star at $V=10$ (see [7]). $V_{\ell}$ is the mode visibility coefficient for mode degrees $\ell$.

Fig. 3 shows the frequency uncertainties computed according to Eq. 1 as a function of the frequency for the reference star. For modes $l=0$ and $l=1$, the uncertainties are of the order of $0.15 \mu \mathrm{Hz}$ for several frequencies around 


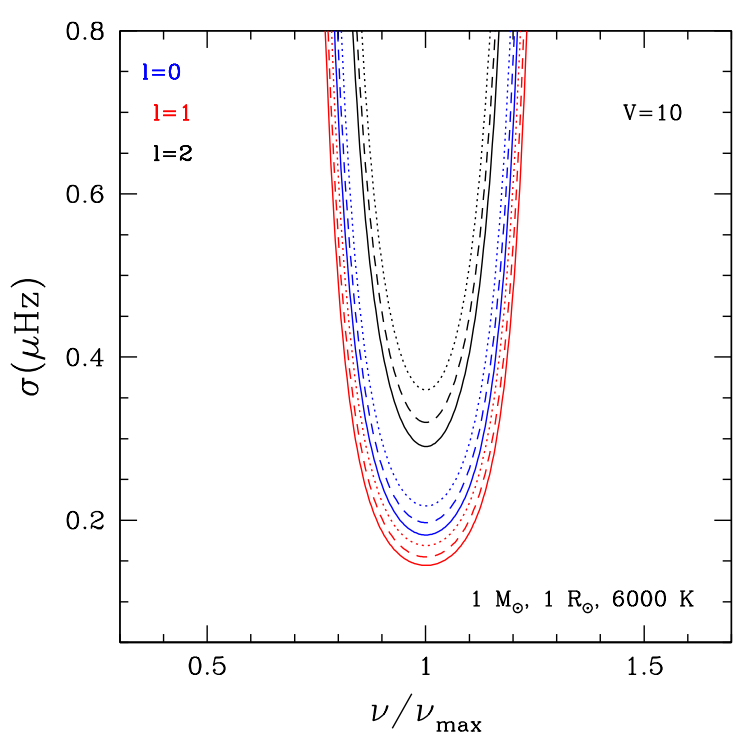

Figure 2: Frequency uncertainties $(\mu \mathrm{Hz})$ for different mode degrees $(l=0,1,2)$ as a function of the frequency normalized to $v_{\max }$. The uncertainty $\sigma$ is computed according to the Libbrecht's relation for the reference star with an apparent magnitude $V=10$, assuming two years of observation and 32 telescopes (solid line), 28 telescopes (dashed line) and 24 telescopes (dotted line).

$v_{\max }$ i.e. satisfying the PLATO specification. For $l=2$, modes the uncertainty amounts to $\sim 0.4 \mu \mathrm{Hz}$.

Step 2 : Let denote the oscillation frequencies, $v_{n, \ell}$, where $n, \ell$ represent the radial order and the degree of the mode, respectively. It is well accepted that the seismic diagnostic $r_{02}$ defined as ([8])

$$
r_{02}(v)=\frac{v_{n, 0}-v_{n-1,2}}{v_{n, 1}-v_{n-1,1}},
$$

provides information on the age of the star [9]. Given the input physics, the computation of stellar models and provides $r_{02}(v)$ and the age of the model. As already stressed by [10], one finds that $r_{02}(v)$ evaluated at $v=v_{\max }$ varies linearly with the age of the stellar model (Fig.4).

$$
r_{02}\left(v_{\max }\right)=c_{0} \text { age } M^{3.4}+c_{1}
$$

with the age in Gyr and the mass in solar unit. The mass dependence of the slope $c_{0}=-6 \cdot 10^{-3} G_{y r^{-1}}$ is negligible. The quantity $c_{1}$ varies with the mass of the star but here we are interested only on the slope $c_{0}$ which is involved in the age uncertainties for the reference star. One can then compute the age uncertainties from the $r_{02}$ uncertainties for the reference star with $\delta$ age $=\delta r_{02} /\left(c_{0}\right)$ while the $r_{02}$ uncertainties are obtained from the observational frequency uncertainties $\delta v_{n, l}$. The validity of the relation between the age uncertainty and the frequency uncertainties was assessed by using the results of the PLATO HH exercises (not shown here).

Fig.4 shows the relative age uncertainty $\delta a g e / a g e$ as a function of the assumed apparent magnitude of the star.

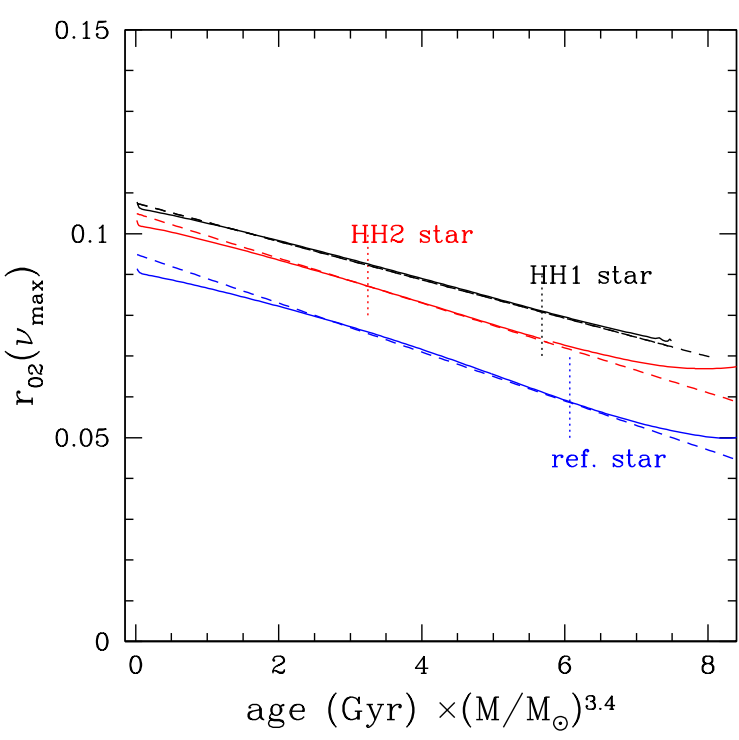

Figure 3: The seismic diagnostic $r_{02}$ evaluated at $v_{\max }$ as a function of age $\times M^{3.4}$ where $M$ is the mass of the model in solar unit and age of the model in Gyr. Each solid curve is the result of a numerical evolution of a stellar model with a given mass. Three cases were considered corresponding to the $\mathrm{HH}$ target case $\mathrm{HH} 1$ $\left(1.18 M_{\odot}\right.$, red line $)$ and $\mathrm{HH} 2\left(1.12 M_{\odot}\right.$, black line $)$ and the reference $\operatorname{star}\left(1 M_{\odot}\right.$, blue line $)$. The stellar models which were input to the $\mathrm{HH}$ exercises and the reference model are located as vertical bars with the same colour than the corresponding lines. The dashed lines are linear fits to the solid lines.

One finds that with an observing time of 2 years, 24 telescopes and the associated NSR, the age specification for the reference star at $V=10$ is easily fulfilled .

However for realistic age uncertainty estimates, we must now take into account the various biases due to stellar modelling.

\subsection{Age systematic error and hare and hound exercices}

The objective of these blind tests was to assess which accuracy/precision is currently obtained in the seismic determination of the age, mass and radius of a star similar- but not identical to the Sun- with the expected Plato data. In the error budget of these $\mathrm{HH}$, were then included the propagation of frequency random errors, the biases due to the number of detected modes which depends on the type of star (mass, effective temperature) and the noise-to-signal ratio, biases due to the optimisation methods used to derive the age from oscillation frequencies, the assumptions made by the hounds for the various physical inputs, the chemical composition and the number and values of the free parameters entering the stellar modelling.

For each hare and hound exercise, the input stellar model and its oscillation frequencies were used to compute a PLATO simulated light curve. This simulated light curve was then analysed to derive the frequencies and their 


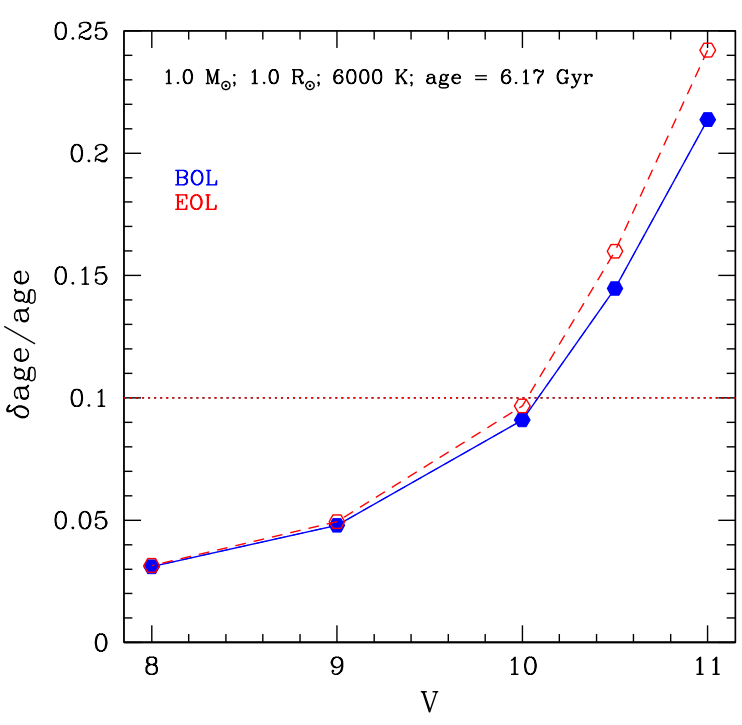

Figure 4: Relative age uncertainties due to the propagation of observational frequency errors as a function of the apparent magnitude of the reference star for 24 telescopes at the beginning (BOL) and end of the mission (EOL).

uncertainties and the parameters identifying the associated oscillation modes (degree, radial order). In a second step, the set of derived frequencies, their uncertainties and mode identification were given to hounds who used them for deriving a set of optimal stellar models, that-is stellar models with frequencies and/or frequency combinations best fitting the corresponding observed quantities. Non seismic constraints such as the effective temperature and optionally the luminosity were also included in the fitting processes. From the set of masses, radii and ages of these optimal models, one derives the estimated mass, radius and age of the 'target star' (i.e. input stellar model) and their uncertainties.

We briefly report here the main results for one such PLATO hare and hound exercise (HH2): the mass and radius of the target (input) stellar model were chosen to be $1.12 M_{\odot}, 1.20 R_{\odot}$. The adopted physical assumptions for the stellar modelling led to an age $=3.44 \mathrm{Gyr}$ (corresponding to a hydrogen central content $X c=0.30$ ) for an effective temperature of $T_{\text {eff }}=6130 \mathrm{~K}$ for this model. We adopted $Y_{i n i}=0.26,(Z / X)_{i n i}=0.019$ for the initial helium abundance in mass and the ratio metallicity over hydrogen.

A more detailed presentation of the expected PLATO seismic performances and results of the PLATO hare and hound exercises will be published a forth coming paper.

\section{Step 1: frequency uncertainties from PLATO simulated light curves}

The transit signature will be remove from the PLATO light curves before any seismic analysis. When computing a simulated light curve, the transit signature is therefore ignored. The stellar activity was assumed to be negligible over the frequency domain of interest for the oscillations of solar-like stars. On the other hand, the impact of the granulation was taken into account.

The simulated light curves were first built in the frequency domain where the oscillation and granulation signals, the target photon noise and the PLATO estimated noise were added together. The stochastically excited oscillating signals were composed of modes with degree $l=0-3$ over a frequency range spanning several radial order around the radial order of the frequency at maximum power. The apparent magnitude of the simulated stars were set to $V=8$ to 11 . The observing time was assumed to be two years and the noise level was set for 32 telescopes (corresponding to a noise-to-signal level of 36 $\mathrm{ppm} / \mathrm{h}$ at $V=11$ ). For the target photon noise, we considered several apparent magnitudes $V=9,10$ and 10.5. Note that the noise level of a $V=11$ star observed with 32 telescopes is the same than for a star with $V=10.6$ with 24 telescopes. Details on the PLATO simulated light curves for seismology can be found in [7].

The simulated light curves were then passed on two groups who carried out independent data analyses: the Birmingham group with B. Chaplin, T. Campante and G. Davies (UK) on one hand and O. Benomar (United Arab Emirates and Germany) on the other hand. They provided sets of 'detected' oscillation frequencies with their uncertainties as well as mode widths and amplitudes with their uncertainties for each simulated light curves (i.e. each assumed apparent magnitude). The degree and radial order were also attributed. As a result, the frequencies are recovered with a good accuracy, indeed the differences between the true frequencies and the frequencies estimated from the data analyses are found to be of the order of $\leq 0.1-0.2 \mu \mathrm{Hz}$ around $v_{\max }$. The frequency uncertainties as estimated by the two groups are of the same order of magnitude. Similar results are found for Kepler solar-like stars [11], [12].

Additional studies by M. Lund (UK, Denmark) investigated the evolution of frequency uncertainties with increasing observing time. Pieces of the $V=10.5 \mathrm{HH} 2$ light curves successively increased by three months periods were analyzed, starting with an observing time of three months and ending with the full 24 months light. As expected, the frequency uncertainty decrease with time as $1 / \sqrt{\text { time }}$. With three months data, the frequency uncertainties are at the level of $0.5-1.5 \mu \mathrm{Hz}$ for $\ell=0,1$ modes and no $l=2$ mode frequency was detected for this specific studied case. The $l=2$ modes near $v_{\max }$ were detected after 6-9 months observations. With 24 months data, the frequency uncertainties are found in the range $0.2-0.4 \mu \mathrm{Hz}$ depending the mode degree, satisfying the PLATO specification for several mode frequencies around $v_{\text {max }}$.

\section{Step 2 : Age systematic errors}

In order to estimate the age systematic error due to uncertainties in stellar modelling, a second step in the HH exercises was performed. The 'observed' frequency sets derived during the first $\mathrm{HH} 2$ step (as described above) were 


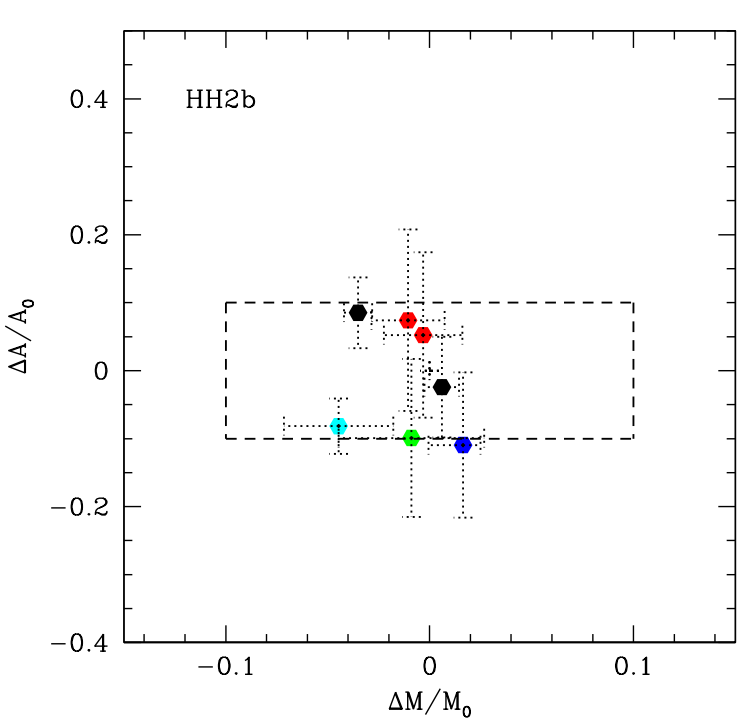

Figure 5: Relative differences between the age of the input model and the ages obtained by the hounds, $\Delta A / A$, obtained for the $\mathrm{HH} 2$ target as a function of the relative differences between the mass of the input model and the masses, $\Delta M / M$. The apparent magnitude is $V=9$, the observing time was set to to years. Each colour corresponds to one hound. The uncertainties as estimated by the hounds are shown as error bar in dotted lines.

given to several 'hounds'. The non seismic observational constraints provided to the hounds were $T_{\text {eff }}=(6100 \pm 80)$ $\mathrm{K} ;[\mathrm{Fe} / \mathrm{H}]=0.04 \pm 0.05 \mathrm{dex} ; \log L / L_{\odot}=0.22 \pm 0.03$. Independent modelling by S. Deheuvels (France), V. SilvaAguirre (Denmark), J. Christensen-Dalsgaard (Denmark), Y. Lebreton (France), O. Creevey(France), A. Mazumdar \& K. Verma (India), I. Roxburgh(UK), M. Bazot (Portugal) used different evolutionary codes with various assumptions for the physical description for the stellar models and their chemical compositions, adopting various seismic constraints and optimisation methods. The hounds provided the mass, radius and age of the $\mathrm{HH} 2$ target and their uncertainties.

Fig. 5 shows the difference between the age of the input model and the age obtained by the hounds, $\Delta A / A$, as obtained for the $\mathrm{HH} 2$ target as a function the difference between the mass of the input model and the masses obtained by each hound, $\Delta M / M$. The target was the $\mathrm{HH} 2$ model with an apparent magnitude $V=9$, the observing time was set to years. The derived values are within $10 \%$ from the exact values. The uncertainties estimated by the hounds are also of the order of $10 \%$.

Similar exercises for the same $\mathrm{HH} 2$ target star but for a magnitude $V=10$ and $V=10.5$ yielded relative uncertainties for the mass in the range: $[0.8-2.5] \%$ and for the age in the range [ $25-27] \%$. For a star at the solar age, the relative age uncertainty then lies the range [ $17-19] \%$ i.e. a few percents up to about 10 percents larger than the PLATO specification.
These results are in full agreement with previous similar studies ([13]; [14], [15] and [16]). For instance, the uncertainties obtained for Kepler host stars (with about four years of observations) yielded $(\Delta A / A)_{\text {stat }} \sim 14 \%+$ several $\%$ (systematics) ([15]).

\section{Conclusion}

The PLATO space mission will detect terrestrial exoplanets up to the habitable zone of solar-type stars and characterize their bulk properties. Several thousands of dwarfs and subgiants with F5 and later spectral type with apparent magnitudes less than 11 will be monitored during long uninterrupted observing runs with a high-precision photometry. Precise and accurate seismic properties will be obtained for a large sample of these stars. Their seismic characterisation will provide masses, radii with respective uncertainties as small as $2 \%, 15 \%$ for stars similar to the reference star with magnitude 10 . Some corrections for systematics errors are needed to reach the PLATO specification for stellar ages $(10 \%$ age uncertainties for the $V=10$ reference star after corrections for biases and systematic effects). For that purpose, improvements in stellar modeling are currently being carried out and will continue in the coming years

The full seismic potential of the stellar part of the core programme of the PLATO mission has not been quantified yet. Indeed a large number of stars will have a noise level much smaller than $34 \mathrm{ppm} / \mathrm{h}$. In addition, many other stars with a noise level $34 \mathrm{ppm} / \mathrm{h}<N S R \leq 36 \mathrm{ppm} / \mathrm{h}$ will be seismically characterized with uncertainties barely higher than for $34 \mathrm{ppm} / \mathrm{h}$.

Studies of massive stars, clusters, red giants will also benefit from the wealth of high quality PLATO data.

More generally, the PLATO legacy database will provide a unique and essential resource for testing our models of planets and stars, their evolution and their interactions.

To become a consortium member, a way is to participate to the PLATO scientific preparation and be part of one workpackage. More details on the stellar part of the PLATO project can be found on the web sites:

$$
\begin{aligned}
& \text { http://www2.warwick.ac.uk/fac/sci/physics/research/ } \\
& \text { astro/plato-science/ } \\
& \text { https://www.ias.u-psud.fr/PLATO_STESCI/PLATO_ } \\
& \text { STESCI_OBJ.html }
\end{aligned}
$$

\section{Acknowledgements}

This work benefited from the financial support provided by the Centre national d'études spatiales (CNES) in the framework of the PLATO project.

\section{References}

[1] Rauer, H. and the PLATO team, Experimental Astronomy 38, 249 (2014)

[2] Goupil, M.J. and the WP120 group, PLATO-LESIAPSPM-TS-01, (issue 1.0) (2015) 
[3] Appourchaux, T., PLATO-IAS-SCI-AN-001, Issue 5 Revision 0, (2016)

[4] Lebreton, Y. and Goupil, M. J. and Montalbán, J., EAS Publications Series 65, 99 (2014b)

[5] Libbrecht, K.G., ApJ 387, 712 (1992)

[6] Toutain, T. and Appourchaux, T., A\&A 289, 649 (1994)

[7] Samadi, R., PLATO-LESIA-PSPM-TN-014, (Issue 1.0 Revision 1.0), (2015)

[8] Roxburgh, I. W., Vorontsov, S. V., A\&A 411, 215 (2003)

[9] Christensen-Dalsgaard, J., Space Research in Stellar Activity and Variability, ed. A. Mangeney \& F. Praderie, 11 (1984)
[10] Appourchaux, T. and Antia, H. M. and Ball, W. et al., A\&A 582, 25 (2015a)

[11] Appourchaux, T. and Chaplin, W. J. and García, R. A. et al., A\&A 543, 54 (2015b)

[12] Davies, G. R. and Silva Aguirre, V. and Bedding, T. R. et al., MNRAS 456, 2183, (2016)

[13] Lebreton, Y. and Goupil, M. J., A\&A 569, 21 (2014a)

[14] Reese, D. R. and Chaplin, W. J. and Davies, G. R. et al., A\&A 592, 14 (2016)

[15] Silva Aguirre, V. and Davies, G. R. and Basu, S. et al., MNRAS 452, 2127, (2015)

[16] Silva Aguirre, V. and Lund, M. N. and Antia, H. M., ApJ 835, 173 (2017) 